\title{
Throat Carriage Rate, Associated Factors, And Antimicrobial Susceptibility Pattern Of Group A Streptococcus Among Healthy School Children In Jigjiga City, Eastern Ethiopia
}

\section{Shamil Barsenga}

Jigjiga University

Habtamu Miteku

Haramaya University

Tewodros Tesfa

Haramaya University

Tadesse Shume ( $\nabla$ tadeshume15@gmail.com )

Haramaya University

\section{Research Article}

Keywords: Throat carriage, Group A Streptococcus, Antimicrobial susceptibility, Children, Jigjiga Ethiopia

Posted Date: February 21st, 2022

DOI: https://doi.org/10.21203/rs.3.rs-1256251/v2

License: (c) (i) This work is licensed under a Creative Commons Attribution 4.0 International License.

Read Full License

Version of Record: A version of this preprint was published at BMC Pediatrics on April 26th, 2022. See the published version at https://doi.org/10.1186/s12887-022-03294-2. 


\section{Abstract}

Background: Group A Streptococcus has been recognized as an important human pathogen and it remains among the top ten causes of mortality from an infectious disease. Group A Streptococcus throat carriage plays an important role in the development of infection and transmission to contacts. In Ethiopia, there is little information about screening of children for Group A Streptococcus carriage.

Objective: This study was aimed to assess the magnitude of throat carriage, associated factors, and antimicrobial susceptibility pattern of Group A Streptococus among healthy school children in Jigjiga city, Eastern Ethiopia from 12 April to 27 May 2021.

Method: A cross-sectional study was conducted among 462 healthy school children in ages ranging from 7 to 14 years. The throat sample was collected using a sterile cotton swab. Identification of Group A Streptococcus was done by colony characterstics, gram staining, catalase negativity, bactricin sensitivity and Pyrrolidonyl arylamidase tests. Antibiotic susceptibility test was done on Muller-Hinton agar containing $5 \%$ sheep blood by modified Kirby-Bauer disc diffusion method. Data on socio-demographic and related characteristics were gathered through interviews using a questionnaire. The data were double-checked, coded, cleaned, and entered onto EpiData Version 3.1 then exported to SPSS version 26.0 for analysis. Bivariate and multivariable logistic regression was used to determine the relationship between outcome and predictor variables. A p-value $<0.05$ was taken as statistically significant.

Results: The overall prevalence of Group A Streptococcus carriage was $10.6 \%$ (95\% Cl; 8.1\% - 13.7\%). Children who live with a family member with a sore throat (AOR=2.51;95\% Cl $1.09-5.73)$, children who live with a large family (AOR=4.64; $95 \% \mathrm{Cl} 1.53-14.1)$, and children who live with non-immediate families (AOR= $3.65 ; 95 \% \mathrm{Cl} 1.39$ - 9.61), showed significant association with Group A Streptococcus carriage. Tetracycline resistance was shown to be high, while antimicrobial drugs such as penicillin, amoxicillin, ceftriaxone, erythromycin, azithromycin, chloramphenicol, and vancomycin were found to be effective. Multi-drug resistance was found in $4.1 \%$ of the isolates.

Conclusion: The present study showed a significant throat carriage of Group A Streptococci/ S. pyogenes in Jigjiga city school children. Family member with a sore throat, having a large family, and living with non-immediate families have all been identified as independent predictors of carriage prevalence. It is recommended that regular screening and surveillance should be conducted in schools.

\section{Introduction}

Group A Streptococcus (GAS) has been an important human pathogen since the early days of modern microbiology, and it is still among the top ten causes of mortality from an infectious disease (1). All Group A streptococcal diseases are most common in settings of poverty, where living conditions promote transmission of the organism, and prevention and treatment programs are less likely to be present or effective (2). 
Group A streptococcus or S. pyogenes can infect people of any age, while children are more likely to be infected (3). Streptococcus pyogenes is responsible for a wide range of clinical symptoms, including impetigo and pharyngitis, as well as more serious disorders such as streptococcal toxic shock syndrome (STSS), endocarditis, and necrotizing fasciitis. Furthermore, autoimmune diseases such as acute poststreptococcal glomerulonephritis and acute rheumatic fever (ARF) can be triggered by recurrent episodes of GAS infection (4).

According to global disease burden figures, WHO ranked GAS as the ninth leading cause of human mortality, with the majority of deaths attributable to invasive GAS infections and RHD (2). The prevalence of GAS disease is estimated at $>18.1$ million cases with an incidence of $>1.78$ million cases per year $(1$, 5). The most common infection caused by GAS is pharyngitis in children between 5 and 15 years of age (6). It is responsible for approximately $15-30 \%$ of cases of pharyngitis in children (7). Failure to eradicate Streptococci from the pharynx occurs in about one-third of non-treated cases, giving rise to the carrier status in those individuals (8). Untreated GAS pharyngitis may trigger acute rheumatic fever and its sequela, rheumatic heart disease, remain important public health problems in low and middle-income countries $(1,9)$.

The existence of the carrier state is reported to be as high as $15-20 \%$ in previous studies (10). Carriers of GAS may represent a potential source for the acquisition of infections for other children and adults (11, 12). According to a review article published by Jane Oliver and colleagues in 2018 , reported $10.5 \%$ and $5.9 \%$ pooled prevalence of GAS carriage in children from high-income countries and children from low/middle-income countries, respectively (13). A few studies were done in Africa on the GAS carriage which ranged around $9.0 \%$ (8). And there is scarcity of information in Ethiopia as well $(14,15)$.

Crowding, limited access to hygiene, inadequate medical care, housing quantity and quality, healthcare access and quality, education, or economic advantage are all risk factors for GAS infection or colonization (carriage) (1).

Even though GAS causes significant problems, there is a lack of data on the epidemiology of GAS carriage in our country. Only a few investigations on GAS have been conducted in previous years (14), particularly it was untouched in Jigjiga, Eastern Ethiopia. Therefore, this study was designed to assess throat carriage prevalence, associated factors, and antimicrobial susceptibility pattern of Group A Streptococci among healthy school children in Jigjiga city, Eastern Ethiopia.

\section{Methods And Materials}

\section{Study Area and Period}

The study was conducted in Jigjiga, a city in the Somali Region of Ethiopia from 12 April to 27 May 2021. Located in the Fafan Zone with $60 \mathrm{~km}$ west of the border with Somalia. Jigjiga is $630 \mathrm{~km}$ east far from the capital city, Addis Ababa There are currently ninety-nine (99) primary schools, seventy-four (74) 
private schools, and twenty-five (25) government schools, with a total of 36,507 students, 20,056 males and 16,451 females (16).

\section{Study Design and population}

A cross-sectional study was conducted. School children from governmental and private primary schools who were randomly selected from twenty schools using a lottery system.

\section{Inclusion and Exclusion Criteria}

All students in selected schools aged 7-14 years old who attend the class during the study period were included in the study. All children on antibiotics for the previous two weeks, as well as those with any signs or symptoms of respiratory diseases such as fever, sore throat, cough, or watery nasal discharge, were excluded from the study.

\section{Sample Size Determination and sampling technique}

A single proportion proportion formula was used to determine the sample size for this quantitative study, considering the following assumptions: A 95\% confidence level, margin of error (0.05) carriage rate among school children, prevalence study of asymptomatic pharyngeal carriage prevalence of $S$. pyogenes, antimicrobial pattern and related risk factors among school children in Hawassa, southern Ethiopia $12.2 \%$ (15). To compute for non-response rate, $10 \%$ of the total sample $=16$ was added. Thus, a total of 178 study subjects were included.

sample size was determined by considering different factor-associated GAS or $S$. pyogenes colonization using double population proportion formula with the assumption of a twosided confidence level of $95 \%$, the margin of error $5 \%$, and power of $80 \%$. Finally, 231 study participants were calculated. So, the sample size for a single population proportion was smaller than the sample size calculated for the second calculated double population proportion, which is a sample size of 231 was used. Because we used a multi-stage sampling method, we introduced a design effect. As a result, 231 was multiplied by 2 , resulting in a sample size of 462 .

\section{Sampling Procedure}

In order to include study participants in the study, a multistage sampling technique was used. There was a total of 99 primary schools (74 private and 25 government). Through a lottery system, twenty (20) schools were chosen at random from both government and private schools. Then the calculated sample size for the study was proportionally allocated to each selected school. A simple random sampling method was used to enroll children. Samples were taken from children whose parents agree to participate until the sample size attend from each selected school. 


\section{Sample Collection and Transportation Methods}

Data on the socio-demographic characteristics of the parents/guardians and children, as well as the children's clinical history, were collected using a structured questionnaire adopted from previous studies $(15,17)$. Two professional nurses administered the questionnaires, and two trained laboratory personnel used cotton swabs to collect a throat sample from a selected child. The throat swab samples were placed in Amie's transport media. Within two hours, the sample was sent in a cold chain to Jigjiga University Sultan Sheik Hassan referral hospital laboratory for investigation.

\section{Laboratory investigation}

The throat sample was cultured on $5 \%$ sheep blood agar plates (Himedia, India) by rolling the swab over a small area of the plate and streaking the sample with a sterile loop, then incubated at $37^{\circ} \mathrm{C}$ with $5 \% \mathrm{CO}_{2}$ atmosphere for 24 hours. A catalase test and gram staining were performed on colonies having ßhemolysis. All catalase-negative and gram-positive cocci were subcultured for 24 hours at $37^{\circ} \mathrm{C}$ on $5 \%$ fresh blood agar plates with a Bacitracin disk in a $5 \% \mathrm{CO}_{2}$ atmosphere to differentiate colonies suspected to be $S$. pyogenes. Any zone of inhibition around the bacitracin disk was a candidate for Pyrrolidonyl arylamidase (PYR) tests, change of color to red /purple was confirmed positive for S. pyogenes. $(18,19)$.

\section{Antimicrobial Susceptibility Testing}

The drug susceptibility test was done by a disk diffusion method by using Muller Hinton Agar (MHA) supplemented with $5 \%$ sheep's blood. Colony suspension was made using normal saline $(0.85 \% \mathrm{NaCl})$ equivalent to $0.5 \%$ McFarland standard from grown overnight colonies (18-24 hours) on sheep blood agar plate.

The suspension was inoculated to an MHA plate with $5 \%$ sheep's blood using a culture swab and incubated at $5 \% \mathrm{CO}_{2}$ for 18 to 24 hours. Drug disks containing penicillin (10 IU), erythromycin $(15 \mathrm{~g})$, azythromycin (15 g), amoxicillin (10 g), chloramphenicol (30 g), ceftriaxone (30 g), vancomycin (30 g), and tetracycline $(10 \mathrm{~g})$ were utilized. The drugs are selected in accordance with the Ethiopian Drug Administration standard treatment guidelines for health centers and Control Authority's and the Clinical Laboratory Standards Institute's (CLSI). The zone of inhibition was measured with a ruler, then recorded and compared to the Clinical and Laboratory Standards Institute (20).

\section{Data Quality Control}

The questionnaire was written in English, then translated into Amharic and Somali, and finally back to English to ensure uniformity. The questionnaire was pre-tested before actual data collection begins. For laboratory testing, the sterility of each batch of produced media was determined by incubating $5 \%$ of the culture media in a $5 \% \mathrm{CO}_{2}$ enriched atmosphere at $37^{\circ} \mathrm{C}$ for 24 hours before using it. Streptococcus pyogenes (ATCC 12696) and Streptococcus agalactae (ATCC 13813) were used as a positive and 
negative control respectively. Quality assurance in antimicrobial susceptibility was done by repeating the selected tests on the same day as the original.

\section{Method of Data Analysis}

The data was entered and coded into the Epi-Data version 3.1 upon creating the questionnaire template. The entered data was cleaned to ensure the validity of all recorded data. The analysis was then carried out using SPSS version 26.0. Descriptive statistics and frequency tables were used to summarize the data. The magnitude of the association between the different variables with the outcome variable was measured by the adjusted odds ratio with a $95 \%$ confidence interval. Bivariate and multivariable logistic regression analysis was made to obtain the odds ratio and the confidence interval of statistical associations. All variables that are significant at $p$-value $<0.25$ in the bivariate analysis were considered for multivarible analysis. The strength of statistical association was measured by adjusted odds ratios and $95 \%$ confidence intervals. Statistical significance was declared at $p<0.05$.

\section{Results}

\section{Sociodemographic characteristics of study participants}

A total of 462 schoolchildren participated in the study, with 244 (52.8\%) of them being female. Two hundred seven students (57.8\%) were from governmental primary schools, while 195 (42.2\%) were from a private primary school in Jigjiga city administration. The study participants' ages range from 7 to 14 , with a mean age was of 10.9 years (SD \pm 1.89 ). The majority (89.4\%) of the participants live in city, and $90.9 \%$ of the students attend school for half-days. Two hundred twenty-nine $(49.6 \%)$ of research participants lived with 6 to 10 family members, while 214 (46.3\%) shared a room with 1 to 3 people. large percentage of $(73.5 \%)$ the parents/guardians of the children were married. Mothers were the primary caregivers in $320(69.3 \%)$ of the cases, and $331(71.6 \%)$ of the respondents had acquired formal education. One hundred forty-three (30.9\%) of the respondants/caregivers were merchants and 108 (23.4\%) were government employees (Table 1). 
Table 1

Sociodemographic characteristics of study participants who participate in the throat carriage prevalence, associated factors, and antimicrobial susceptibility pattern of Group A Streptococcus among healthy school children in Jigjiga city, Eastern Ethiopia from 12 April to 27 May 2021

\section{Variables}

Frequency

Percentage (\%)

\section{Socio-demographic characteristics of the child}

\begin{tabular}{|c|c|c|c|}
\hline \multirow[t]{2}{*}{ Age of the child } & $7-10$ & 199 & 43.1 \\
\hline & $11-14$ & 263 & 56.9 \\
\hline \multirow[t]{2}{*}{ Sex of the child } & Male & 218 & 47.2 \\
\hline & Female & 244 & 52.8 \\
\hline \multirow[t]{2}{*}{ Residence } & In the town & 413 & 89.4 \\
\hline & Outskirt & 49 & 10.6 \\
\hline \multirow[t]{2}{*}{ Duration of child stay in school } & Half-day & 420 & 90.9 \\
\hline & Full day & 42 & 9.1 \\
\hline \multirow[t]{3}{*}{ Family Size } & $1-5$ & 142 & 30.7 \\
\hline & $6-10$ & 229 & 49.6 \\
\hline & $11-15$ & 91 & 19.7 \\
\hline \multirow[t]{3}{*}{ Room Sharing } & $1-3$ & 214 & 46.3 \\
\hline & $4-6$ & 199 & 43.1 \\
\hline & $7-9$ & 49 & 10.6 \\
\hline \multicolumn{4}{|c|}{ The social condition of the parent/guardian of the child } \\
\hline \multirow[t]{4}{*}{ Marital status } & Married & 340 & 73.5 \\
\hline & Divorced & 35 & 7.6 \\
\hline & Widowed & 17 & 3.7 \\
\hline & Single & 70 & 15.2 \\
\hline \multirow[t]{3}{*}{ Care givers } & Mother & 320 & 69.3 \\
\hline & Father & 109 & 23.6 \\
\hline & Non-immediate family ${ }^{1}$ & 33 & 7.1 \\
\hline
\end{tabular}

${ }^{1}$ Grandparent, aunt, and/or uncle

${ }^{2}$ Primary (1-8), secondary (9-12), tertiary (diploma and above), and/or degree and above 


\begin{tabular}{|c|c|c|c|}
\hline Variables & & Frequency & Percentage (\%) \\
\hline \multirow[t]{2}{*}{ Educational status } & Unable to read and write & 131 & 28.4 \\
\hline & Received formal education ${ }^{2}$ & 331 & 71.6 \\
\hline \multirow[t]{5}{*}{ Occupation } & Searching for Job & 25 & 5.4 \\
\hline & Stay home parent & 106 & 22.9 \\
\hline & Merchant & 143 & 31.0 \\
\hline & Private employee & 80 & 17.3 \\
\hline & Government employee & 108 & 23.4 \\
\hline \multirow[t]{3}{*}{ Monthly income (ETB) } & $250-1000$ & 108 & 23.4 \\
\hline & $1000-3000$ & 56 & 28.6 \\
\hline & $>3000$ & 222 & 48.1 \\
\hline \multicolumn{4}{|c|}{${ }^{1}$ Grandparent, aunt, and/or uncle } \\
\hline \multicolumn{4}{|c|}{2 Primary (1-8), secondary (9-12), tertiary (diploma and above), and/or degree and above } \\
\hline
\end{tabular}

\section{Clinical history of the study participants}

The majority $(76 \%)$ of the study participants had no history of hospitalization in the previous 5 years and $87.9 \%$ of family member had no history sore throat. Nearly, half of the parents $47.6 \%$ claimed that they had never given antibiotics to their children.

\section{Prevalence of S. pyogenes}

Among 462 school children, 49 (10.6\%) (95\%CL; 8.1\% - 13.7\%) were confirmed to have S. pyogenes in their throats.

\section{Analysis for factors associated with GAS colonization}

Children who lived with a family member who had a sore throat were twice likely to have $S$. pyogenes in their throat compared with those who lived with no family member who had a sore throat $(A O R=2.51$; $95 \% \mathrm{Cl} 1.09-5.73$;). Children living in families with more than 11 members were four times more likely carrying S. pyogenes compared to children living in families with less members (AOR=4.64; $95 \% \mathrm{Cl} 1.53-$ 14.1). Children living with non-immediate families were more than three times more likely to have $S$. pyogenes compare to children living with their mothers ( $\mathrm{AOR}=3.65 ; 95 \% \mathrm{Cl} 1.39-9.61$ (Table 2) 
Table 2

Bivariate and multivariable analysis of participating on the throat carriage prevalence, associated factors, and antimicrobial susceptibility pattern of Group A Streptococci among healthy school children in Jigjiga city, Eastern Ethiopia from 12 April to 27 May 2021

\begin{tabular}{|c|c|c|c|c|c|c|}
\hline \multirow[t]{2}{*}{ Variables } & \multicolumn{2}{|c|}{ Culture result } & \multirow{2}{*}{$\begin{array}{l}\text { COR } \\
(95 \% \mathrm{C} . \\
\text { I) }\end{array}$} & \multirow{2}{*}{$\begin{array}{l}\text { p- } \\
\text { value } \\
\text { (a) }\end{array}$} & \multirow{2}{*}{$\begin{array}{l}\text { AOR } \\
(95 \% \\
\text { C.I) }\end{array}$} & \multirow{2}{*}{$\begin{array}{l}\text { p- } \\
\text { value(b) }\end{array}$} \\
\hline & $\begin{array}{l}\text { GAS } \\
\text { negative }\end{array}$ & $\begin{array}{l}\text { GAS } \\
\text { positive }\end{array}$ & & & & \\
\hline & $\begin{array}{l}n=413 \\
(89.4 \%)\end{array}$ & $\begin{array}{l}n=49 \\
(10.6 \%)\end{array}$ & & & & \\
\hline
\end{tabular}

\section{Socio-demographic characteristics}

\begin{tabular}{|c|c|c|c|c|c|}
\hline \multirow[t]{2}{*}{ Age of the child } & $7-10$ & 181(91.0) & 18(9.0) & $\begin{array}{l}0.74 \\
(0.40 \\
-1.37)\end{array}$ & 0.3 \\
\hline & $11-14$ & $\begin{array}{l}232 \\
(88.2)\end{array}$ & $\begin{array}{l}31 \\
(11.8)\end{array}$ & 1 & \\
\hline \multirow[t]{2}{*}{ Sex of the child } & Male & 197(90.4) & $21(9.6)$ & 1 & \\
\hline & Female & $\begin{array}{l}216 \\
(88.5)\end{array}$ & $\begin{array}{l}28 \\
(11.5)\end{array}$ & $\begin{array}{l}1.22 \\
(0.67- \\
2.21)\end{array}$ & 0.5 \\
\hline
\end{tabular}

\section{Parental/Guardian Social condition}

\begin{tabular}{|c|c|c|c|c|c|c|c|}
\hline \multirow[t]{4}{*}{ Marital Status } & Single & $63(90)$ & $7(10)$ & $\begin{array}{l}0.97 \\
(0.41- \\
2.28)\end{array}$ & 0.94 & & \\
\hline & Widowed & $13(76.4)$ & $4(23.5)$ & $\begin{array}{l}2.68 \\
(0.83- \\
8.67)\end{array}$ & 0.10 & & \\
\hline & Divorced & 32 (91.4) & $3(8.6)$ & $\begin{array}{l}0.82 \\
(0.24- \\
2.80)\end{array}$ & 0.75 & & \\
\hline & Married & $\begin{array}{l}305 \\
(89.7)\end{array}$ & $\begin{array}{l}35 \\
(10.3)\end{array}$ & 1 & & & \\
\hline Caregivers & $\begin{array}{l}\text { Non- } \\
\text { immediate } \\
\text { family }{ }^{1}\end{array}$ & $24(72.7)$ & $9(27.3)$ & $\begin{array}{l}3.15 \\
(1.36- \\
7.34)\end{array}$ & 0.008 & $\begin{array}{l}3.65 \\
(1.39 \\
-9.61)\end{array}$ & $0.009 *$ \\
\hline
\end{tabular}

*Statistically significant;

${ }^{1}$ Grandparent, aunt, and/or uncle ${ }^{2}$ Primary (1-8), secondary (9-12), tertiary (diploma and above), and/or degree and above;

AOR=Adjusted Odds Ratio, COR=Crude Odds Ratio 


\begin{tabular}{|c|c|c|c|c|c|c|c|}
\hline \multirow[t]{3}{*}{ Variables } & & \multicolumn{2}{|c|}{ Culture result } & \multirow{3}{*}{$\begin{array}{l}\text { COR } \\
(95 \% \mathrm{C} .\end{array}$} & \multirow{3}{*}{$\begin{array}{l}\text { p- } \\
\text { value } \\
\text { (a) }\end{array}$} & \multirow{3}{*}{$\begin{array}{l}\text { AOR } \\
(95 \% \\
\text { C.I) }\end{array}$} & \multirow{3}{*}{$\begin{array}{l}\mathrm{p}- \\
\text { value(b) }\end{array}$} \\
\hline & & $\begin{array}{l}\text { GAS } \\
\text { negative }\end{array}$ & $\begin{array}{l}\text { GAS } \\
\text { positive }\end{array}$ & & & & \\
\hline & & $\begin{array}{l}n=413 \\
(89.4 \%)\end{array}$ & $\begin{array}{l}n=49 \\
(10.6 \%)\end{array}$ & & & & \\
\hline & Father & $\begin{array}{l}104 \\
(95.4)\end{array}$ & $5(4.6)$ & $\begin{array}{l}0.49 \\
(0.20- \\
1.20)\end{array}$ & 0.12 & $\begin{array}{l}0.50 \\
(0.18 \\
- \\
1.36)\end{array}$ & 0.174 \\
\hline & Mother & $\begin{array}{l}285 \\
(89.1)\end{array}$ & $\begin{array}{l}35 \\
(10.9)\end{array}$ & 1 & & 1 & \\
\hline \multirow[t]{2}{*}{ Education status } & $\begin{array}{l}\text { Unable to } \\
\text { read and } \\
\text { write }\end{array}$ & $\begin{array}{l}111 \\
(84.7)\end{array}$ & $\begin{array}{l}20 \\
(15.3)\end{array}$ & $\begin{array}{l}1.88 \\
(1.03- \\
3.45)\end{array}$ & 0.043 & $\begin{array}{l}1.11 \\
(0.53 \\
2.30)\end{array}$ & 0.78 \\
\hline & $\begin{array}{l}\text { Recived } \\
\text { formal } \\
\text { education } 2\end{array}$ & $\begin{array}{l}302 \\
(91.2)\end{array}$ & $29(8.8)$ & 1 & & & \\
\hline \multirow[t]{5}{*}{ Occupation } & $\begin{array}{l}\text { Searching for } \\
\text { Job }\end{array}$ & $23(92.0)$ & $2(8.0)$ & $\begin{array}{l}0.69 \\
(0.15- \\
3.33)\end{array}$ & 0.65 & & \\
\hline & $\begin{array}{l}\text { Stay home } \\
\text { parent }\end{array}$ & $88(83.0)$ & $\begin{array}{l}18 \\
(17.0)\end{array}$ & $\begin{array}{l}1.64 \\
(0.75- \\
3.59)\end{array}$ & 0.22 & & \\
\hline & Merchant & $\begin{array}{l}129 \\
(90.2)\end{array}$ & $14(9.8)$ & $\begin{array}{l}0.87 \\
(0.38- \\
1.96)\end{array}$ & 0.73 & & \\
\hline & $\begin{array}{l}\text { Private } \\
\text { employee }\end{array}$ & 77 (96.3) & $3(3.8)$ & $\begin{array}{l}0.31 \\
(0.08- \\
1.14)\end{array}$ & 0.08 & & \\
\hline & $\begin{array}{l}\text { Government } \\
\text { employee }\end{array}$ & $96(88.9)$ & $\begin{array}{l}12 \\
(11.1)\end{array}$ & 1 & & & \\
\hline $\begin{array}{l}\text { Monthly income } \\
\text { (ETB) }\end{array}$ & $250-1000$ & $86(79.6)$ & $\begin{array}{l}22 \\
(20.4)\end{array}$ & $\begin{array}{l}3.08 \\
(1.56- \\
6.09)\end{array}$ & 0.001 & $\begin{array}{l}1.87 \\
(0.85 \\
4.15)\end{array}$ & 0.21 \\
\hline
\end{tabular}

*Statistically significant;

${ }^{1}$ Grandparent, aunt, and/or uncle ${ }^{2}$ Primary (1-8), secondary (9-12), tertiary (diploma and above), and/or degree and above;

AOR=Adjusted Odds Ratio, COR=Crude Odds Ratio 


\begin{tabular}{|c|c|c|c|c|c|c|c|}
\hline \multirow[t]{3}{*}{ Variables } & & \multicolumn{2}{|c|}{ Culture result } & \multirow{3}{*}{$\begin{array}{l}\text { COR } \\
(95 \% \mathrm{C} . \\
\text { l) }\end{array}$} & \multirow{3}{*}{$\begin{array}{l}\text { p- } \\
\text { value } \\
\text { (a) }\end{array}$} & \multirow{3}{*}{$\begin{array}{l}\text { AOR } \\
(95 \% \\
\text { C.I) }\end{array}$} & \multirow{3}{*}{$\begin{array}{l}\text { p- } \\
\text { value(b) }\end{array}$} \\
\hline & & $\begin{array}{l}\text { GAS } \\
\text { negative }\end{array}$ & $\begin{array}{l}\text { GAS } \\
\text { positive }\end{array}$ & & & & \\
\hline & & $\begin{array}{l}n=413 \\
(89.4 \%)\end{array}$ & $\begin{array}{l}n=49 \\
(10.6 \%)\end{array}$ & & & & \\
\hline & $1000-3000$ & $\begin{array}{l}122 \\
(92.4)\end{array}$ & $10(7.6)$ & $\begin{array}{l}0.98 \\
(0.44- \\
2.23)\end{array}$ & 0.99 & $\begin{array}{l}0.79 \\
(0.33 \\
- \\
1.91)\end{array}$ & 0.61 \\
\hline & $>3000$ & $\begin{array}{l}205 \\
(92.3)\end{array}$ & $17(7.7)$ & 1 & & 1 & \\
\hline \multicolumn{8}{|c|}{ Child living condition } \\
\hline \multirow[t]{3}{*}{ Family size } & $1-5$ & $\begin{array}{l}134 \\
(94.4)\end{array}$ & $8(5.6)$ & 1 & & 1 & \\
\hline & 6- 10 & $\begin{array}{l}211 \\
(92.1)\end{array}$ & $18(7.9)$ & $\begin{array}{l}1.43 \\
(0.60- \\
3.38)\end{array}$ & 0.42 & $\begin{array}{l}1.41 \\
(0.53 \\
-3.72)\end{array}$ & 0.493 \\
\hline & $11-15$ & $68(74.7)$ & $\begin{array}{l}23 \\
(25.3)\end{array}$ & $\begin{array}{l}5.67 \\
(2.40- \\
13.3)\end{array}$ & 0.000 & $\begin{array}{l}4.64 \\
(1.53 \\
- \\
14.1)\end{array}$ & $0.007^{\star}$ \\
\hline \multirow[t]{3}{*}{ Room sharing } & $1-3$ & $\begin{array}{l}201 \\
(93.9)\end{array}$ & $13(6.1)$ & 1 & & 1 & \\
\hline & $4-6$ & $\begin{array}{l}174 \\
(87.4)\end{array}$ & $\begin{array}{l}25 \\
(12.6)\end{array}$ & $\begin{array}{l}2.22 \\
(1.10- \\
4.48)\end{array}$ & 0.025 & $\begin{array}{l}1.48 \\
(0.64 \\
- \\
3.41)\end{array}$ & 0.356 \\
\hline & $7-9$ & 38 (87.8) & $11(22.4)$ & $\begin{array}{l}4.48 \\
(1.87- \\
10.7)\end{array}$ & 0.01 & $\begin{array}{l}1.89 \\
(0.59 \\
- \\
6.06)\end{array}$ & 0.280 \\
\hline \multirow[t]{2}{*}{ Residence } & In the town & $\begin{array}{l}372 \\
(90.1)\end{array}$ & $41(9.9)$ & 1 & & & \\
\hline & Outskirt & $41(83.7)$ & $8(16.3)$ & $\begin{array}{l}1.77 \\
(0.78- \\
4.03)\end{array}$ & 0.174 & & \\
\hline
\end{tabular}

*Statistically significant;

${ }^{1}$ Grandparent, aunt, and/or uncle ${ }^{2}$ Primary (1-8), secondary (9-12), tertiary (diploma and above), and/or degree and above;

AOR=Adjusted Odds Ratio, COR=Crude Odds Ratio 


\begin{tabular}{|c|c|c|c|c|c|c|c|}
\hline \multirow[t]{3}{*}{ Variables } & & \multicolumn{2}{|c|}{ Culture result } & \multirow{3}{*}{$\begin{array}{l}\text { COR } \\
(95 \% \mathrm{C} . \\
\text { I) }\end{array}$} & \multirow{3}{*}{$\begin{array}{l}\text { p- } \\
\text { value } \\
\text { (a) }\end{array}$} & \multirow{3}{*}{$\begin{array}{l}\text { AOR } \\
\text { (95\% } \\
\text { C.l) }\end{array}$} & \multirow{3}{*}{$\begin{array}{l}\text { p- } \\
\text { value(b) }\end{array}$} \\
\hline & & $\begin{array}{l}\text { GAS } \\
\text { negative }\end{array}$ & $\begin{array}{l}\text { GAS } \\
\text { positive }\end{array}$ & & & & \\
\hline & & $\begin{array}{l}n=413 \\
(89.4 \%)\end{array}$ & $\begin{array}{l}n=49 \\
(10.6 \%)\end{array}$ & & & & \\
\hline \multirow[t]{2}{*}{$\begin{array}{l}\text { Duration of child } \\
\text { stay in school }\end{array}$} & Half-day & $\begin{array}{l}375 \\
(89.3)\end{array}$ & $\begin{array}{l}45 \\
(10.7)\end{array}$ & 1 & & & \\
\hline & Full day & $38(90.5)$ & $4(9.5)$ & $\begin{array}{l}1.14 \\
(0.39- \\
3.34)\end{array}$ & 0.811 & & \\
\hline \multicolumn{8}{|c|}{ Clinical History of the child } \\
\hline \multirow{4}{*}{$\begin{array}{l}\text { Earlier antibiotic } \\
\text { use }\end{array}$} & $1-6$ months & $61(89.7)$ & $7(10.3)$ & 1 & & & \\
\hline & $\begin{array}{l}6-12 \\
\text { months }\end{array}$ & $73(90.1)$ & $8(9.9)$ & $\begin{array}{l}0.84 \\
(0.37- \\
1.87)\end{array}$ & 0.662 & & \\
\hline & $>12$ month & $84(90.3)$ & $9(9.7)$ & $\begin{array}{l}0.86 \\
(0.37- \\
1.98)\end{array}$ & 0.714 & & \\
\hline & No & $\begin{array}{l}195 \\
(88.6)\end{array}$ & $\begin{array}{l}25 \\
(11.4)\end{array}$ & $\begin{array}{l}0.89 \\
(0.37- \\
2.17)\end{array}$ & 0.806 & & \\
\hline \multirow[t]{2}{*}{$\begin{array}{l}\text { Hospitalization } \\
\text { history (<5 } \\
\text { years) }\end{array}$} & Yes & $96(86.5)$ & $\begin{array}{l}15 \\
(13.5)\end{array}$ & $\begin{array}{l}1.46 \\
(0.76- \\
2.79)\end{array}$ & 0.256 & & \\
\hline & No & $\begin{array}{l}317 \\
(90.3)\end{array}$ & $34(9.7)$ & 1 & & 1 & \\
\hline \multirow[t]{2}{*}{$\begin{array}{l}\text { Family member } \\
\text { with a sore throat }\end{array}$} & Yes & $45(80.4)$ & $\begin{array}{l}11 \\
(19.6)\end{array}$ & $\begin{array}{l}2.37 \\
(1.13- \\
4.95)\end{array}$ & 0.022 & $\begin{array}{l}2.51 \\
(1.09 \\
5.73)\end{array}$ & $0.029 *$ \\
\hline & No & $368(90.6)$ & $38(9.4)$ & 1 & & 1 & \\
\hline $\begin{array}{l}\text { Sore throat } \\
\text { (within the last } \\
30 \text { days) }\end{array}$ & Yes & 79 (83.2) & $\begin{array}{l}16 \\
(16.8)\end{array}$ & $\begin{array}{l}2.05 \\
(1.08- \\
3.91)\end{array}$ & 0.03 & $\begin{array}{l}1.86 \\
(0.90 \\
- \\
3.85)\end{array}$ & 0.093 \\
\hline
\end{tabular}

*Statistically significant;

${ }^{1}$ Grandparent, aunt, and/or uncle ${ }^{2}$ Primary (1-8), secondary (9-12), tertiary (diploma and above), and/or degree and above;

AOR=Adjusted Odds Ratio, COR=Crude Odds Ratio 


\begin{tabular}{|c|c|c|c|c|c|c|}
\hline \multirow[t]{3}{*}{ Variables } & \multicolumn{2}{|c|}{ Culture result } & \multirow{3}{*}{$\begin{array}{l}\text { COR } \\
(95 \% \mathrm{C} . \\
\text { l) }\end{array}$} & \multirow{3}{*}{$\begin{array}{l}\text { p- } \\
\text { value } \\
\text { (a) }\end{array}$} & \multirow{3}{*}{$\begin{array}{l}\text { AOR } \\
(95 \% \\
\text { C.I) }\end{array}$} & \multirow{3}{*}{$\begin{array}{l}\text { p- } \\
\text { value(b) }\end{array}$} \\
\hline & $\begin{array}{l}\text { GAS } \\
\text { negative }\end{array}$ & $\begin{array}{l}\text { GAS } \\
\text { positive }\end{array}$ & & & & \\
\hline & $\begin{array}{l}n=413 \\
(89.4 \%)\end{array}$ & $\begin{array}{l}n=49 \\
(10.6 \%)\end{array}$ & & & & \\
\hline No & $\begin{array}{l}334 \\
(91.1)\end{array}$ & $33(8.9)$ & 1 & & & \\
\hline \multicolumn{7}{|l|}{ *Statistically significant; } \\
\hline \multicolumn{7}{|c|}{$\begin{array}{l}{ }^{1} \text { Grandparent, aunt, and/or uncle }{ }^{2} \text { Primary (1-8), secondary (9-12), tertiary (diploma and above), } \\
\text { and/or degree and above; }\end{array}$} \\
\hline \multicolumn{7}{|c|}{ AOR=Adjusted Odds Ratio, $\mathrm{COR}=$ Crude Odds Ratio } \\
\hline
\end{tabular}

\section{Antibiotic Susceptibility testing}

To determine the antimicrobial susceptibility patterns of isolates, those 49 bacterial isolates were tested for eight different antimicrobials. Majority of them were susceptible to Amoxicillin 44 (89.8\%), Azithromycin 45 (91.8\%)), Ceftriaxone 47 (95.9\%), Chloramphenicol 46 (93.9\%), Erythromycin 46 (93.9\%), Penicillin 49 (100\%), and Vancomycin 45 (91.8\%). At the same time, more than half of the bacterial isolates were resistant to Tetracycline 26 (53.1\%) (Table 3). Moreover, two (4.1\%) of the 49 isolates were drug-resistant to three drugs, particularly Tetracycline, Amoxacillin, and Eryomycin/Vancomycin. 
Table 3

Antimicrobial susceptibility pattern of isolates from children who participate in the study for throat carriage prevalence, associated factors, and antimicrobial susceptibility pattern of Group A Streptococcus among healthy school children in Jigjiga city, Eastern Ethiopia from 12 April to 27 May 2021

\begin{tabular}{|lllll|}
\hline Antibiotics & Total isolates & Susceptible & Intermediate & Resistant \\
\hline Amoxicillin & 49 & Number (\%) & Number (\%) & Number (\%) \\
\hline Azithromycin & 49 & $44(89.8 \%)$ & $3(6.1 \%)$ & $2(4.1)$ \\
\hline Ceftriaxone & 49 & $45(91.8 \%)$ & $4(8.2 \%)$ & - \\
\hline Chloramphenicol & 49 & $47(95.9 \%)$ & $2(4.1 \%)$ & - \\
\hline Erythromycin & 49 & $46(93.9 \%)$ & $3(6.1 \%)$ & - \\
\hline Penicillin & 49 & $46(93.9 \%)$ & $2(4.1 \%)$ & $1(2.0 \%)$ \\
\hline Tetracycline & 49 & $49(100 \%)$ & - & - \\
\hline Vancomyin & 49 & $18(36.7 \%)$ & $5(10.2 \%)$ & $26(53.1 \%)$ \\
\hline
\end{tabular}

\section{Discussion}

Group A Streptococcus has been a major human pathogen, since the dawn of modern microbiology and it is still one of the top ten causes of death from infectious diseases (1). In the present study the overall pharyngeal carriage rate of $S$. pyogenes was $10.6 \%$. (95\% Cl; $8.1 \%-13.7 \%)$. A carrier rate comparable to ours has been recorded in Ethiopia (9.7\% and 12.2\%, respectively) $(14,15)$, United Arab Emirates (10\%) (21), Nepal (10.8\%) (22), and Yemen (12.8\%) (23). However, the prevalence was lower than those reported in Uganda (16.0\%) (24), Egypt (16\%) (25), India (23.1\%) (17), Argentina (14.2\%) (26) and Brazil (14.0\%) (27). Our results showed a higher carriage rate than Gabon's 5.8\% (27) and Nepal's 5.0\% (28). The possible reasons for the discrepancy include hygiene, awareness of the route of microbial disease transmission, sample size, seasonal change, and geography, and socio-demographic diversity $(15,23-$ 25).

Children who lived with a family member who had a sore throat were twice as likely to have Group A Streptococcus than children who did not have a primary case or sore throat in their household. In Melbourne, Australia, researchers discovered that when a primary case is present, the likelihood of subsequent infection within a family increases by 1.8 times (29). In addition, according to a study conducted in Nepal, one out of every five children is affected when a family member has already been infected. (22).

Children from families with more than eleven members had a four-fold greater chance of carrying GAS than children from families with fewer members. Similarly, research in Hawassa found that children living 
in families with more than five members were more than 10 times more likely to be carriers of $S$. pyogenes than children living in families with fewer members. (15). According to another study from Iraq, the carrier rate among children living in homes with more than six individuals is two times higher than among children living in families with fewer members (30). According to the findings of these investigations, there is a substantial link between the carrier rate and the number of family members. The explanation for this could be that an increase in the number of family members increases the danger of infection, including GAS.

Unlike a study by Asrat Anja (15), in this study Children living with non-immediate families were more than three times more likely to have Group A Streptococci / S. pyogenes than children living with their immediate family. Crowding and poor hygiene, therefore, increases the chance of the transmission of $S$. pyogenes (31). As reported in Ethiopia (14) and other regions of the world, there was no significant statistical connection between GAS colonization rate and gender or age in this study $(17,23,25)$.

All GAS isolates were sensitive to penicillin in our study. The same high activity of penicillin had been reported in many countries, namely Ethiopia $(14,15,32)$, Uganda (24), India (17), Nepal $(22,28)$ and Argentina (26). Our findings reveal that amoxicillin (89.8\%) and ceftriaxone (95.9\%) have slightly reduced sensitivity against $S$. pyogenes when compared to recently published publications in Senegal and Ethiopia $(15,33)$.

For patients allergic to penicillin, erythromycin and other macrolides were suggested as first-line alternatives (6). The isolates displayed the same level of sensitivity to erythromycin and azithromycin (93.9\%). The isolates also showed $93.9 \%$ and $91.8 \%$ sensitivity to chloramphenicol and vancomycin, respectively. A comparable result was reported from Ethiopia $(14,15,32)$ and other parts of the world $(17$, $24-26,33,34)$.

Tetracycline resistance was found to be relatively high (53.1\%) in our study. Tetracycline resistance at high levels in GAS isolates may reflect the country's widespread usage of the antibiotic. This finding was comparable with high resistance rates (40-100\%) reported in Ethiopia $(14,15)$, Uganda (24), Egypt (25), Senegal (33), and Argentina (26). About 2(4.1\%) of isolated GAS isolates were showed multiple drug resistant. A comparable result was reported from in Hawwasa, Ethiopia. (15).

\section{Limitation of the study}

Since the study was a cross-sectional study conducted over a short period, the impact of environmental or seasonal factors on variations in prevalence could not be determined. In addition, this study was unable to performed ASO titer due to a lack of resources.

\section{Conclusion}

The present study showed a significant throat carriage of GAS in the Jigjiga city school children population. Children with a sore throat in the family, children from a large family, and children from non- 
immediate families have all been identified as independent predictors of carriage prevalence. Penicillin, amoxicillin, ceftriaxone, chloramphenicol, erythromycin, azithromycin, and vancomycin remain very sensitive against $S$. pyogenes Isolates.

The current research highlights the importance of conducting school surveys regularly. In order to reduce the streptococcal disease burden in the community, well-designed longitudinal research and the adoption of effective public health strategies are also required.

\section{Abbreviations}

AOR $\quad$ Adjusted Odds Ratio

ASO Antitreptolysin 0

AST Antibiotic Susceptibility Test

CLSI Clinical Laboratory Standard Institute

COR $\quad$ Crude Odds Ratio

GAS Group A Streptococcus

MHA Mueller Hinton Agar

\section{Declarations}

\section{Ethical approval and consent}

The study was conducted out after receiving ethical approval from Haramaya University College of Health Science and Medical Sciences Institutional Health Research Ethics Review Committee (IHRERC). This study was conducted in accordance with the Declaration of Helsinki. Each child's parent/guardian has been sufficiently informed of the study's purpose and the importance of their participation by the data collectors and/or the investigator. Written, Informed, voluntary, and signed consent were taken from all parents/guardians and assent were taken from the child before commencing the study. All parents/guardians were consulted about the benefit of the treatment by a pediatrician who works at Jigjiga University Sultan Shiek Hassen Referral Hospital and the drugs were given free of charge.

\section{Consent for publication}

Not applicable.

\section{Availability of data and materials}


The data sets generated during and/or analyzed during the current study are available from the corresponding authors on reasonable request.

\section{Conflicts of interests}

There were no conflicts of interest.

\section{Funding statement}

This research data collection finance was covered by Haramaya university postgraduate directorate.

\section{Authors' contributions}

All authors made a significant contribution to the work reported, whether that is in the conception, study design, execution, acquisition of data, analysis, and interpretation, or in all these areas; took part in drafting, revising or critically reviewing the article; gave final approval of the version to be published; have agreed on the journal to which the article has been submitted; and agree to be accountable for all aspects of the work.

\section{Acknowledgments}

We acknowledged Haramaya University Colleges of Health and Medical Sciences Institutional Health Research Ethical Review Committee for giving the ethical clearance. We also thank study participants and all individuals who have in one way or another contributed to the completion of this research.

\section{References}

1. Ralph A, Carapetis J. Group A Streptococcal Diseases and Their Global Burden. Current topics in microbiology and immunology. 2012;368.

2. World Health Organization. The current evidence for the burden of group A streptococcal diseases. World Health Organization; 2005.

3. Efstratiou A, Lamagni T. Epidemiology of Streptococcus pyogenes. In: Ferretti JJ, Stevens DL, Fischetti VA, editors. Streptococcus pyogenes: Basic Biology to Clinical Manifestations. Oklahoma City (OK): University of Oklahoma Health Sciences Center (c) The University of Oklahoma Health Sciences Center.; 2016.

4. Carapetis JR, Steer AC, Mulholland EK, Weber M. The global burden of group A streptococcal diseases. The Lancet Infectious diseases. 2005;5(11):685-94.

5. Barth DD, Moloi A, Mayosi BM, Engel ME. Prevalence of group A Streptococcal infection in Africa to inform GAS vaccines for rheumatic heart disease: A systematic review and meta-analysis. International Journal of Cardiology. 2020;307:200-8.

6. Bisno A, Gerber M, Gwaltney J, Kaplan E, Schwartz R. Infectious Diseases Society of America. Practice guidelines for the diagnosis and management of group A streptococcal pharyngitis. Clin 
Infect Dis. 2002;35(2):113-25.

7. Platt M, Vicario S, Marx J. Rosen's emergency medicine: Concepts and clinical practice 7th ed Philadelphia. Pa: Mosby Elsevier. 2010;57.

8. Engel ME, Mayosi BM. Clinical and epidemiological aspects of streptococcus pyogenes pharyngitis and carriage in Africa: streptococcus pyogenes in Africa. SA Heart. 2013;10(2):434-9.

9. Matthys $J$. There are still problems in identifying who will develop complications of sore throat in primary care. BMJ (Clinical research ed). 2014;348:g299.

10. Henningham A, Barnett TC, Maamary PG, Walker MJ. Pathogenesis of group A streptococcal infections. Discovery medicine. 2012;13(72):329-42.

11. Bessen DE. Population biology of the human restricted pathogen, Streptococcus pyogenes. Infection, Genetics and Evolution. 2009;9(4):581-93.

12. Sanyahumbi AS, Colquhoun S, Wyber R, Carapetis JR. Global disease burden of group A Streptococcus. Streptococcus pyogenes: basic biology to clinical manifestations [Internet]: University of Oklahoma Health Sciences Center; 2016.

13. Oliver J, Malliya Wadu E, Pierse N, Moreland NJ, Williamson DA, Baker MG. Group A Streptococcus pharyngitis and pharyngeal carriage: A meta-analysis. PLOS Neglected Tropical Diseases. 2018;12(3):e0006335.

14. Abdissa A, Asrat D, Kronvall G, Shitu B, Achiko D, Zeidan M, et al. Throat carriage rate and antimicrobial susceptibility pattern of group A Streptococci (GAS) in healthy Ethiopian school children. Ethiop Med J. 2011;49(2):125-30.

15. Anja A, Beyene G, Daka D. Asymptomatic pharyngeal carriage rate of Streptococcus pyogenes, its associated factors and antibiotic susceptibility pattern among school children in Hawassa town, southern Ethiopia. BMC research notes. 2019;12(1):564.

16. Jigjiga City Administration Education Bureau. General school information. Jigjiga, Ethiopia: Jigjiga City Administration Education Bureau,; 2020.

17. Mukundan A, Vijayakumar S. Pharyngeal carriage of Group A streptococci among school children. Journal of International Medicine and Dentistry. 2017;4(1):18-26.

18. Brooks GF, Butel JS, Morse SA. Jawetz, Melnick, \& Adelberg's medical microbiology: Lange Medical Books/McGraw-Hill, Medical Pub. Division; 2004.

19. Cheesbrough M. District laboratory practice in tropical countries, part 2: Cambridge university press; 2006.

20. Clinical and Laboratory Standards Institute. Performance Standards for Antimicrobial Susceptibility Testing. CLSI supplement: Wayne, PA:; 2020.

21. Ali Al Shamisi FH. The Prevalence of Streptococcus Pyogenes and its emm Gene Types among School Children in Al Ain, UAE. 2016.

22. Prajapati A, Rai S, Mukhiya R, Karki A. Study on carrier rate of Streptococcus pyogenes among the school children and antimicrobial susceptibility pattern of isolates. Nepal Medical College journal: 
NMCJ. 2012;14:169-71.

23. Othman AM, Assayaghi RM, Al-Shami HZ, Saif-Ali R. Asymptomatic carriage of Streptococcus pyogenes among school children in Sana'a city, Yemen. BMC Research Notes. 2019;12(1):339.

24. Nayiga I, Okello E, Lwabi P, Ndeezi G. Prevalence of group a streptococcus pharyngeal carriage and clinical manifestations in school children aged 5-15 yrs in Wakiso District, Uganda. BMC Infectious Diseases. 2017;17(1):248.

25. Abd El-Ghany SM, Abdelmaksoud AA, Saber SM, Abd El Hamid DH. Group A beta-hemolytic streptococcal pharyngitis and carriage rate among Egyptian children: a case-control study. Annals of Saudi medicine. 2015;35(5):377-82.

26. Delpech G, Sparo M, Baldaccini B, Pourcel G, Lissarrague S, Allende LG. Throat carriage rate and antimicrobial resistance of Streptococcus pyogenes in rural children in Argentina. Journal of Preventive Medicine and Public Health. 2017;50(2):127.

27. Bélard S, Toepfner N, Arnold B, Alabi AS, Berner R. $\beta$-hemolytic streptococcal throat carriage and tonsillopharyngitis: a cross-sectional prevalence study in Gabon, Central Africa. Infection. 2015;43(2):177-83.

28. Manandhar A, Shah Y, Shrestha J. Study on the prevalence of beta haemolytic Streptococcus among school children. Journal of Nepal Paediatric Society. 2013;33(1):45-7.

29. Danchin MH, Rogers S, Kelpie L, Selvaraj G, Curtis N, Carlin JB, et al. Burden of acute sore throat and group A streptococcal pharyngitis in school-aged children and their families in Australia. Pediatrics. 2007;120(5):950-7.

30. Saleh MMS. CARRIAGE STATE OF GABHS AMONG YEMENI SCHOOLCHILDREN AND THE UPPER LIMIT OF NORMAL FOR ASO IN DIFFERENT POPULATION GROUPS. Iraqi Journal of Science. 2010;51(1):63-70.

31. Faruq Q, Rashid A, Ahmed J, Waiz A, Haque K, Rouf M, et al. Prevalence of streptococcal sorethroat in the school children of Dhaka. Bangladesh Medical Research Council bulletin. 1995;21(3):87-94.

32. Zegeye N, Asrat D, Woldeamanuel Y, Habte A, Gedlu E, Tønjum T, et al. Throat culture positivity rate and antibiotic susceptibility pattern of beta-hemolytic streptococci in children on secondary prophylaxis for rheumatic heart disease. BMC Infectious Diseases. 2016;16(1):510.

33. Camara M, Dieng A, Boye CSB. Antibiotic susceptibility of streptococcus pyogenes isolated from respiratory tract infections in dakar, senegal. Microbiology insights. 2013;6:MBI. S12996.

34. Bobia AA, Blaj OA, Oancea D, lulia-Cristina B, Radu-Vasile B, Delia-loana $H$, et al. The prevalence of beta hemolytic Streptococcus in a Children's Tertiary Care Hospital in Timisoara. Central European Journal of Clinical Research. 2019;2(1):73-8. 